\title{
Hypo- $q$-norms on cartesian products of algebras of bounded linear operators on Hilbert spaces
}

\author{
S.S. Dragomir ${ }^{1,2}$ \\ ${ }^{1}$ Mathematics, College of Engineering 83 Science \\ Victoria University, Melbourne City 8001, Australia \\ ${ }^{2}$ DST-NRF Centre of Excellence in the Mathematical and Statistical Sciences \\ School of Computer Science \& Applied Mathematics \\ University of the Witwatersrand, Johannesburg 2050, South Africa \\ sever.dragomir@vu.edu.au, http://rgmia.org/dragomir
}

Abstract: In this paper we introduce the hypo- $q$-norms on a Cartesian product of algebras of bounded linear operators on Hilbert spaces. A representation of these norms in terms of inner products, the equivalence with the $q$-norms on a Cartesian product and some reverse inequalities obtained via the scalar reverses of Cauchy-Buniakowski-Schwarz inequality are also given. Several bounds for the norms $\delta_{p}, \vartheta_{p}$ and the real norms $\eta_{r, p}$ and $\theta_{r, p}$ are provided as well.

Key words: Hilbert spaces, bounded linear operators, operator norm and numerical radius, $n$-tuple of operators, operator inequalities.

AMS Subject Class. (2010): 46C05, 26D15.

\section{INTRODUCTION}

In [13, the author has introduced the following norm on the Cartesian product $B^{(n)}(H):=B(H) \times \cdots \times B(H)$, where $B(H)$ denotes the Banach algebra of all bounded linear operators defined on the complex Hilbert space $H$ :

$$
\left\|\left(T_{1}, \ldots, T_{n}\right)\right\|_{n, e}:=\sup _{\left(\lambda_{1}, \ldots, \lambda_{n}\right) \in \mathbb{B}_{n}}\left\|\lambda_{1} T_{1}+\cdots+\lambda_{n} T_{n}\right\|,
$$

where $\left(T_{1}, \ldots, T_{n}\right) \in B^{(n)}(H)$ and

$$
\mathbb{B}_{n}:=\left\{\left(\lambda_{1}, \ldots, \lambda_{n}\right) \in \mathbb{C}^{n}: \sum_{i=1}^{n}\left|\lambda_{i}\right|^{2} \leq 1\right\}
$$

is the Euclidean closed ball in $\mathbb{C}^{n}$. It is clear that $\|\cdot\|_{n, e}$ is a norm on $B^{(n)}(H)$ and for any $\left(T_{1}, \ldots, T_{n}\right) \in B^{(n)}(H)$ we have

$$
\left\|\left(T_{1}, \ldots, T_{n}\right)\right\|_{n, e}=\left\|\left(T_{1}^{*}, \ldots, T_{n}^{*}\right)\right\|_{n, e},
$$


where $T_{i}^{*}$ is the adjoint operator of $T_{i}, i \in\{1, \ldots, n\}$.

It has been shown in [13] that the following inequality holds true:

$$
\frac{1}{\sqrt{n}}\left\|\sum_{j=1}^{n} T_{j} T_{j}^{*}\right\|^{\frac{1}{2}} \leq\left\|\left(T_{1}, \ldots, T_{n}\right)\right\|_{n, e} \leq\left\|\sum_{j=1}^{n} T_{j} T_{j}^{*}\right\|^{\frac{1}{2}}
$$

for any $n$-tuple $\left(T_{1}, \ldots, T_{n}\right) \in B^{(n)}(H)$ and the constants $\frac{1}{\sqrt{n}}$ and 1 are best possible.

In the same paper [13] the author has introduced the Euclidean operator radius of an $n$-tuple of operators $\left(T_{1}, \ldots, T_{n}\right)$ by

$$
w_{n, e}\left(T_{1}, \ldots, T_{n}\right):=\sup _{\|x\|=1}\left(\sum_{j=1}^{n}\left|\left\langle T_{j} x, x\right\rangle\right|^{2}\right)^{\frac{1}{2}}
$$

and proved that $w_{n, e}(\cdot)$ is a norm on $B^{(n)}(H)$ and satisfies the double inequality:

$$
\frac{1}{2}\left\|\left(T_{1}, \ldots, T_{n}\right)\right\|_{n, e} \leq w_{n, e}\left(T_{1}, \ldots, T_{n}\right) \leq\left\|\left(T_{1}, \ldots, T_{n}\right)\right\|_{n, e}
$$

for each $n$-tuple $\left(T_{1}, \ldots, T_{n}\right) \in B^{(n)}(H)$.

As pointed out in [13], the Euclidean numerical radius also satisfies the double inequality:

$$
\frac{1}{2 \sqrt{n}}\left\|\sum_{j=1}^{n} T_{j} T_{j}^{*}\right\|^{\frac{1}{2}} \leq w_{n, e}\left(T_{1}, \ldots, T_{n}\right) \leq\left\|\sum_{j=1}^{n} T_{j} T_{j}^{*}\right\|^{\frac{1}{2}}
$$

for any $\left(T_{1}, \ldots, T_{n}\right) \in B^{(n)}(H)$ and the constants $\frac{1}{2 \sqrt{n}}$ and 1 are best possible.

Now, let $(E,\|\cdot\|)$ be a normed linear space over the complex number field $\mathbb{C}$. On $\mathbb{C}^{n}$ endowed with the canonical linear structure we consider a norm $\|\cdot\|_{n}$. As an example of such norms we should mention the usual $p$-norms

$$
\|\lambda\|_{n, p}:= \begin{cases}\max \left\{\left|\lambda_{1}\right|, \ldots,\left|\lambda_{n}\right|\right\} & \text { if } p=\infty, \\ \left(\sum_{k=1}^{n}\left|\lambda_{k}\right|^{p}\right)^{\frac{1}{p}} & \text { if } p \in[1, \infty) .\end{cases}
$$

The Euclidean norm is obtained for $p=2$, i.e.,

$$
\|\lambda\|_{n, 2}:=\left(\sum_{k=1}^{n}\left|\lambda_{k}\right|^{2}\right)^{\frac{1}{2}}
$$


It is well known that on $E^{n}:=E \times \cdots \times E$ endowed with the canonical linear structure we can define the following $p$-norms:

$$
\|x\|_{n, p}:= \begin{cases}\max \left\{\left|x_{1}\right|, \ldots,\left|x_{n}\right|\right\} & \text { if } p=\infty, \\ \left(\sum_{k=1}^{n}\left|x_{k}\right|^{p}\right)^{\frac{1}{p}} & \text { if } p \in[1, \infty) .\end{cases}
$$

where $x=\left(x_{1}, \ldots, x_{n}\right) \in E^{n}$.

Following the paper [5], for a given norm $\|\cdot\|_{n}$ on $\mathbb{C}^{n}$, we define the functional $\|\cdot\|_{h, n}: E^{n} \rightarrow[0, \infty)$ by

$$
\|x\|_{h, n}:=\sup _{\|\lambda\|_{n} \leq 1}\left\|\sum_{j=1}^{n} \lambda_{j} x_{j}\right\|
$$

where $x=\left(x_{1}, \ldots, x_{n}\right) \in E^{n}$ and $\lambda=\left(\lambda_{1}, \ldots, \lambda_{n}\right) \in \mathbb{C}^{n}$.

It is easy to see that [5]:

(i) $\|x\|_{h, n} \geq 0$ for any $x \in E^{n}$,

(ii) $\|x+y\|_{h, n} \leq\|x\|_{h, n}+\|y\|_{h, n}$ for any $x, y \in E^{n}$,

(iii) $\|\alpha x\|_{h, n}=|\alpha|\|x\|_{h, n}$ for each $\alpha \in \mathbb{C}$ and $x \in E^{n}$,

and therefore $\|\cdot\|_{h, n}$ is a semi-norm on $E^{n}$. This will be called the hypo-seminorm generated by the norm $\|\cdot\|_{n}$ on $E^{n}$.

We observe that $\|x\|_{h, n}=0$ if and only if $\sum_{j=1}^{n} \lambda_{j} x_{j}=0$ for any $\left(\lambda_{1}, \ldots, \lambda_{n}\right) \in B\left(\|\cdot\|_{n}\right)$. If there exists $\lambda_{1}^{0}, \ldots, \lambda_{n}^{0} \neq 0$ such that $\left(\lambda_{1}^{0}, 0, \ldots, 0\right)$, $\left(0, \lambda_{2}^{0}, \ldots, 0\right), \ldots,\left(0,0, \ldots, \lambda_{n}^{0}\right) \in B\left(\|\cdot\|_{n}\right)$ then the semi-norm generated by $\|\cdot\|_{n}$ is a norm on $E^{n}$.

If $p \in[1, \infty]$ and we consider the $p$-norms $\|\cdot\|_{n, p}$ on $\mathbb{C}^{n}$, then we can define the following hypo-q-norms on $E^{n}$ :

$$
\|x\|_{h, n, q}:=\sup _{\|\lambda\|_{n, p} \leq 1}\left\|\sum_{j=1}^{n} \lambda_{j} x_{j}\right\|,
$$

with $q \in[1, \infty]$. If $p=1$, then $q=\infty$; if $p=\infty$, then $q=1$; if $p \in(1, \infty)$, then $\frac{1}{p}+\frac{1}{q}=1$.

For $p=2$, we have the hypo-Euclidean norm on $E^{n}$, i.e.,

$$
\|x\|_{h, n, e}:=\sup _{\|\lambda\|_{n, 2} \leq 1}\left\|\sum_{j=1}^{n} \lambda_{j} x_{j}\right\| .
$$


If we consider now $E=B(H)$ endowed with the operator norm $\|\cdot\|$, then we can obtain the following hypo-q-norms on $B^{(n)}(H)$

$$
\left\|\left(T_{1}, \ldots, T_{n}\right)\right\|_{h, n, q}:=\sup _{\|\lambda\|_{n, p} \leq 1}\left\|\sum_{j=1}^{n} \lambda_{j} T_{j}\right\| \quad \text { where } p, q \in[1, \infty],
$$

with the convention that if $p=1, q=\infty$, if $p=\infty, q=1$ and if $p>1$, then $\frac{1}{p}+\frac{1}{q}=1$.

For $p=2$ we obtain the hypo-Euclidian norm $\|(\cdot, \ldots, \cdot)\|_{n, e}$ defined in 1.2 .

If we consider now $E=B(H)$ endowed with the operator numerical radius $w(\cdot)$, which is a norm on $B(H)$, then we can obtain the following hypo-qnumerical radius of $\left(T_{1}, \ldots, T_{n}\right) \in B^{(n)}(H)$ defined by

$$
w_{h, n, q}\left(T_{1}, \ldots, T_{n}\right):=\sup _{\|\lambda\|_{n, p} \leq 1} w\left(\sum_{j=1}^{n} \lambda_{j} T_{j}\right) \quad \text { with } p, q \in[1, \infty],
$$

with the convention that if $p=1, q=\infty$, if $p=\infty, q=1$ and if $p>1$, then $\frac{1}{p}+\frac{1}{q}=1$.

For $p=2$ we obtain the hypo-Euclidian norm

$$
w_{h, n, e}\left(T_{1}, \ldots, T_{n}\right):=\sup _{\|\lambda\|_{n, 2} \leq 1} w\left(\sum_{j=1}^{n} \lambda_{j} T_{j}\right)
$$

and will show further that it coincides with the Euclidean operator radius of an $n$-tuple of operators $\left(T_{1}, \ldots, T_{n}\right)$ defined in 1.4 .

Using the fundamental inequality between the operator norm and numerical radius $w(T) \leq\|T\| \leq 2 w(T)$ for $T \in B(H)$ we have

$$
w\left(\sum_{j=1}^{n} \lambda_{j} T_{j}\right) \leq\left\|\sum_{j=1}^{n} \lambda_{j} T_{j}\right\| \leq 2 w\left(\sum_{j=1}^{n} \lambda_{j} T_{j}\right)
$$

for any $\left(T_{1}, \ldots, T_{n}\right) \in B^{(n)}(H)$ and any $\lambda=\left(\lambda_{1}, \ldots, \lambda_{n}\right) \in \mathbb{C}^{n}$. By taking the supremum over $\lambda$ with $\|\lambda\|_{n, p} \leq 1$ we get

$$
w_{h, n, q}\left(T_{1}, \ldots, T_{n}\right) \leq\left\|\left(T_{1}, \ldots, T_{n}\right)\right\|_{h, n, q} \leq 2 w_{h, n, q}\left(T_{1}, \ldots, T_{n}\right)
$$

with the convention that if $p=1, q=\infty$, if $p=\infty, q=1$ and if $p>1$, then $\frac{1}{p}+\frac{1}{q}=1$. 
For $p=q=2$ we recapture the inequality 1.5 .

In 2012, 8] (see also [9, 10]) the author have introduced the concept of $s$ - $q$-numerical radius of an $n$-tuple of operators $\left(T_{1}, \ldots, T_{n}\right)$ for $q \geq 1$ as

$$
w_{s, q}\left(T_{1}, \ldots, T_{n}\right):=\sup _{\|x\|=1}\left(\sum_{j=1}^{n}\left|\left\langle T_{j} x, x\right\rangle\right|^{q}\right)^{1 / q}
$$

and established various inequalities of interest. For more recent results see also [12, 14].

In the same paper [8] we also introduced the concept of $s$ - $q$-norm of an $n$-tuple of operators $\left(T_{1}, \ldots, T_{n}\right)$ for $q \geq 1$ as

$$
\left\|\left(T_{1}, \ldots, T_{n}\right)\right\|_{s, q}:=\sup _{\|x\|=\|y\|=1}\left(\sum_{j=1}^{n}\left|\left\langle T_{j} x, y\right\rangle\right|^{q}\right)^{1 / q} .
$$

In [8], [9] and [10], by utilising Kato's inequality [11]

$$
|\langle T x, y\rangle|^{2} \leq\left\langle|T|^{2 \alpha} x, x\right\rangle\left\langle\left|T^{*}\right|^{2(1-\alpha)} y, y\right\rangle
$$

for any $x, y \in H, \alpha \in[0,1]$, where "absolute value" operator of $A$ is defined by $\|A\|:=\sqrt{A^{*} A}$, the authors have obtained several inequalities for the $s$ - $q$ numerical radius and $s$-q-norm.

In this paper we investigate the connections between these norms and establish some fundamental inequalities of interest in multivariate operator theory.

\section{Representation Results}

We start with the following lemma:

Lemma 1. Let $\beta=\left(\beta_{1}, \ldots, \beta_{n}\right) \in \mathbb{C}^{n}$.

(i) If $p, q>1$ and $\frac{1}{p}+\frac{1}{q}=1$, then

$$
\sup _{\|\alpha\|_{n, p} \leq 1}\left|\sum_{j=1}^{n} \alpha_{j} \beta_{j}\right|=\|\beta\|_{n, q} .
$$

In particular,

$$
\sup _{\|\alpha\|_{n, 2} \leq 1}\left|\sum_{j=1}^{n} \alpha_{j} \beta_{j}\right|=\|\beta\|_{n, 2}
$$


(ii) We have

$$
\sup _{\|\alpha\|_{n, \infty} \leq 1}\left|\sum_{j=1}^{n} \alpha_{j} \beta_{j}\right|=\|\beta\|_{n, 1} \text { and } \sup _{\|\alpha\|_{n, 1} \leq 1}\left|\sum_{j=1}^{n} \alpha_{j} \beta_{j}\right|=\|\beta\|_{n, \infty} .
$$

Proof. (i) Using Hölder's discrete inequality for $p, q>1$ and $\frac{1}{p}+\frac{1}{q}=1$ we have

$$
\left|\sum_{j=1}^{n} \alpha_{j} \beta_{j}\right| \leq\left(\sum_{j=1}^{n}\left|\alpha_{j}\right|^{p}\right)^{1 / p}\left(\sum_{j=1}^{n}\left|\beta_{j}\right|^{q}\right)^{1 / q}
$$

which implies that

$$
\sup _{\|\alpha\|_{n, p} \leq 1}\left|\sum_{j=1}^{n} \alpha_{j} \beta_{j}\right| \leq\|\beta\|_{n, q}
$$

where $\alpha=\left(\alpha_{1}, \ldots, \alpha_{n}\right)$ and $\beta=\left(\beta_{1}, \ldots, \beta_{n}\right)$ are $n$-tuples of complex numbers.

For $\left(\beta_{1}, \ldots, \beta_{n}\right) \neq 0$, consider $\alpha=\left(\alpha_{1}, \ldots, \alpha_{n}\right)$ with

$$
\alpha_{j}:=\frac{\overline{\beta_{j}}\left|\beta_{j}\right|^{q-2}}{\left(\sum_{k=1}^{n}\left|\beta_{k}\right|^{q}\right)^{1 / p}}
$$

for those $j$ for which $\beta_{j} \neq 0$ and $\alpha_{j}=0$, for the rest.

We observe that

$$
\begin{aligned}
\left|\sum_{j=1}^{n} \alpha_{j} \beta_{j}\right| & =\left|\sum_{j=1}^{n} \frac{\overline{\beta_{j}}\left|\beta_{j}\right|^{q-2}}{\left(\sum_{k=1}^{n}\left|\beta_{k}\right|^{q}\right)^{1 / p}} \beta_{j}\right|=\frac{\sum_{j=1}^{n}\left|\beta_{j}\right|^{q}}{\left(\sum_{k=1}^{n}\left|\beta_{k}\right|^{q}\right)^{1 / p}} \\
& =\left(\sum_{j=1}^{n}\left|\beta_{j}\right|^{q}\right)^{1 / q}=\|\beta\|_{n, q}
\end{aligned}
$$

and

$$
\begin{aligned}
\|\alpha\|_{n, p}^{p} & =\sum_{j=1}^{n}\left|\alpha_{j}\right|^{p}=\sum_{j=1}^{n} \frac{\left.\left.\left|\overline{\beta_{j}}\right| \beta_{j}\right|^{q-2}\right|^{p}}{\left(\sum_{k=1}^{n}\left|\beta_{k}\right|^{q}\right)}=\sum_{j=1}^{n} \frac{\left(\left|\beta_{j}\right|^{q-1}\right)^{p}}{\left(\sum_{k=1}^{n}\left|\beta_{k}\right|^{q}\right)} \\
& =\sum_{j=1}^{n} \frac{\left|\beta_{j}\right|^{q p-p}}{\left(\sum_{k=1}^{n}\left|\beta_{k}\right|^{q}\right)}=\sum_{j=1}^{n} \frac{\left|\beta_{j}\right|^{q}}{\left(\sum_{k=1}^{n}\left|\beta_{k}\right|^{q}\right)}=1 .
\end{aligned}
$$

Therefore, by (2.4) we have the representation (2.1). 
(ii) Using the properties of the modulus, we have

$$
\left|\sum_{j=1}^{n} \alpha_{j} \beta_{j}\right| \leq \max _{j \in\{1, \ldots, n\}}\left|\alpha_{j}\right| \sum_{j=1}^{n}\left|\beta_{j}\right|,
$$

which implies that

$$
\sup _{\|\alpha\|_{n, \infty} \leq 1}\left|\sum_{j=1}^{n} \alpha_{j} \beta_{j}\right| \leq\|\beta\|_{n, 1},
$$

where $\alpha=\left(\alpha_{1}, \ldots, \alpha_{n}\right)$ and $\beta=\left(\beta_{1}, \ldots, \beta_{n}\right)$.

For $\left(\beta_{1}, \ldots, \beta_{n}\right) \neq 0$, consider $\alpha=\left(\alpha_{1}, \ldots, \alpha_{n}\right)$ with $\alpha_{j}:=\frac{\overline{\beta_{j}}}{\left|\beta_{j}\right|}$ for those $j$ for which $\beta_{j} \neq 0$ and $\alpha_{j}=0$, for the rest.

We have

$$
\left|\sum_{j=1}^{n} \alpha_{j} \beta_{j}\right|=\left|\sum_{j=1}^{n} \frac{\overline{\beta_{j}}}{\left|\beta_{j}\right|} \beta_{j}\right|=\sum_{j=1}^{n}\left|\beta_{j}\right|=\|\beta\|_{n, 1}
$$

and

$$
\|\alpha\|_{n, \infty}=\max _{j \in\{1, \ldots, n\}}\left|\alpha_{j}\right|=\max _{j \in\{1, \ldots, n\}}\left|\frac{\overline{\beta_{j}}}{\left|\beta_{j}\right|}\right|=1
$$

and by 2.5 we get the first representation in $(2.3)$.

Moreover, we have

$$
\left|\sum_{j=1}^{n} \alpha_{j} \beta_{j}\right| \leq \sum_{j=1}^{n}\left|\alpha_{j}\right| \max _{j \in\{1, \ldots, n\}}\left|\beta_{j}\right|,
$$

which implies that

$$
\sup _{\|\alpha\|_{n, 1} \leq 1}\left|\sum_{j=1}^{n} \alpha_{j} \beta_{j}\right| \leq\|\beta\|_{n, \infty}
$$

where $\alpha=\left(\alpha_{1}, \ldots, \alpha_{n}\right)$ and $\beta=\left(\beta_{1}, \ldots, \beta_{n}\right)$.

For $\left(\beta_{1}, \ldots, \beta_{n}\right) \neq 0$, let $j_{0} \in\{1, \ldots, n\}$ such that

$$
\|\beta\|_{\infty}=\max _{j \in\{1, \ldots, n\}}\left|\beta_{j}\right|=\left|\beta_{j_{0}}\right| .
$$

Consider $\alpha=\left(\alpha_{1}, \ldots, \alpha_{n}\right)$ with $\alpha_{j_{0}}=\frac{\overline{\beta_{j_{0}}}}{\left|\beta_{j_{0}}\right|}$ and $\alpha_{j}=0$ for $j \neq j_{0}$. For this choice we get

$$
\sum_{j=1}^{n}\left|\alpha_{j}\right|=\frac{\left|\overline{\beta_{0}}\right|}{\left|\beta_{j_{0}}\right|}=1 \quad \text { and } \quad\left|\sum_{j=1}^{n} \alpha_{j} \beta_{j}\right|=\left|\frac{\overline{\beta_{j_{0}}}}{\left|\beta_{j_{0}}\right|} \beta_{j_{0}}\right|=\left|\beta_{j_{0}}\right|=\|\beta\|_{n, \infty},
$$


therefore by (2.6) we obtain the second representation in (4).

Theorem 2. Let $\left(T_{1}, \ldots, T_{n}\right) \in B^{(n)}(H)$ and $x, y \in H$, then for $p, q>1$ and $\frac{1}{p}+\frac{1}{q}=1$ we have

$$
\sup _{\|\alpha\|_{n, p} \leq 1}\left|\left\langle\left(\sum_{j=1}^{n} \alpha_{j} T_{j}\right) x, y\right\rangle\right|=\left(\sum_{j=1}^{n}\left|\left\langle T_{j} x, y\right\rangle\right|^{q}\right)^{1 / q}
$$

and in particular

$$
\sup _{\|\alpha\|_{n, 2} \leq 1}\left|\left\langle\left(\sum_{j=1}^{n} \alpha_{j} T_{j}\right) x, y\right\rangle\right|=\left(\sum_{j=1}^{n}\left|\left\langle T_{j} x, y\right\rangle\right|^{2}\right)^{1 / 2} .
$$

We also have

$$
\sup _{\|\alpha\|_{n, \infty} \leq 1}\left|\left\langle\left(\sum_{j=1}^{n} \alpha_{j} T_{j}\right) x, y\right\rangle\right|=\sum_{j=1}^{n}\left|\left\langle T_{j} x, y\right\rangle\right|
$$

and

$$
\sup _{\|\alpha\|_{n, 1} \leq 1}\left|\left\langle\left(\sum_{j=1}^{n} \alpha_{j} T_{j}\right) x, y\right\rangle\right|=\max _{j \in\{1, \ldots, n\}}\left\{\left|\left\langle T_{j} x, y\right\rangle\right|\right\} .
$$

Proof. If we take $\beta=\left(\left\langle T_{1} x, y\right\rangle, \ldots,\left\langle T_{n} x, y\right\rangle\right) \in \mathbb{C}^{n}$ in 2.1), then we get

$$
\begin{aligned}
\left(\sum_{j=1}^{n}\left|\left\langle T_{j} x, y\right\rangle\right|^{q}\right)^{1 / q} & =\|\beta\|_{n, q}=\sup _{\|\alpha\|_{p} \leq 1}\left|\sum_{j=1}^{n} \alpha_{j} \beta_{j}\right| \\
& =\sup _{\|\alpha\|_{n, p} \leq 1}\left|\sum_{j=1}^{n} \alpha_{j}\left\langle T_{j} x, y\right\rangle\right|=\sup _{\|\alpha\|_{n, p} \leq 1}\left|\left\langle\sum_{j=1}^{n} \alpha_{j} T_{j} x, y\right\rangle\right|,
\end{aligned}
$$

which proves (2.7).

The equalities 2.9 and 2.10 follow by $(2.3)$.

Corollary 3. Let $\left(T_{1}, \ldots, T_{n}\right) \in B^{(n)}(H)$ and $x \in H$, then for $p, q>1$ and $\frac{1}{p}+\frac{1}{q}=1$ we have

$$
\sup _{\|\alpha\|_{n, p} \leq 1}\left|\left\langle\left(\sum_{j=1}^{n} \alpha_{j} T_{j}\right) x, x\right\rangle\right|=\left(\sum_{j=1}^{n}\left|\left\langle T_{j} x, x\right\rangle\right|^{q}\right)^{1 / q}
$$


and, in particular

$$
\sup _{\|\alpha\|_{n, 2} \leq 1}\left|\left\langle\left(\sum_{j=1}^{n} \alpha_{j} T_{j}\right) x, x\right\rangle\right|=\left(\sum_{j=1}^{n}\left|\left\langle T_{j} x, x\right\rangle\right|^{2}\right)^{1 / 2} .
$$

We also have

$$
\sup _{\|\alpha\|_{n, \infty} \leq 1}\left|\left\langle\left(\sum_{j=1}^{n} \alpha_{j} T_{j}\right) x, x\right\rangle\right|=\sum_{j=1}^{n}\left|\left\langle T_{j} x, x\right\rangle\right|
$$

and

$$
\sup _{\|\alpha\|_{n, 1} \leq 1}\left|\left\langle\left(\sum_{j=1}^{n} \alpha_{j} T_{j}\right) x, x\right\rangle\right|=\max _{j \in\{1, \ldots, n\}}\left\{\left|\left\langle T_{j} x, x\right\rangle\right|\right\} .
$$

Corollary 4. Let $\left(T_{1}, \ldots, T_{n}\right) \in B^{(n)}(H)$ and $x \in H$, then for $p, q>1$ and $\frac{1}{p}+\frac{1}{q}=1$ we have

$$
\sup _{\|\alpha\|_{n, p} \leq 1}\left\|\sum_{j=1}^{n} \alpha_{j} T_{j} x\right\|=\sup _{\|y\|=1}\left(\sum_{j=1}^{n}\left|\left\langle T_{j} x, y\right\rangle\right|^{q}\right)^{1 / q}
$$

and in particular

$$
\sup _{\|\alpha\|_{n, 2} \leq 1}\left\|\sum_{j=1}^{n} \alpha_{j} T_{j} x\right\|=\sup _{\|y\|=1}\left(\sum_{j=1}^{n}\left|\left\langle T_{j} x, y\right\rangle\right|^{2}\right)^{1 / 2} .
$$

We also have

$$
\sup _{\|\alpha\|_{n, \infty} \leq 1}\left\|\sum_{j=1}^{n} \alpha_{j} T_{j} x\right\|=\sup _{\|y\|=1} \sum_{j=1}^{n}\left|\left\langle T_{j} x, y\right\rangle\right|
$$

and

$$
\sup _{\|\alpha\|_{n, 1} \leq 1}\left\|\sum_{j=1}^{n} \alpha_{j} T_{j} x\right\|=\max _{j \in\{1, \ldots, n\}}\left\{\left\|T_{j} x\right\|\right\} .
$$

Proof. By the properties of inner product, we have for any $u \in H, u \neq 0$ that

$$
\|u\|=\sup _{\|y\|=1}|\langle u, y\rangle|
$$


Let $x \in H$, then by taking the supremum over $\|y\|=1$ in (2.7) we get for $p, q>1$ with $\frac{1}{p}+\frac{1}{q}=1$ that

$$
\begin{aligned}
\sup _{\|y\|=1}\left(\sum_{j=1}^{n}\left|\left\langle T_{j} x, y\right\rangle\right|^{q}\right)^{1 / q} & =\sup _{\|y\|=1}\left(\sup _{\|\alpha\|_{n, p} \leq 1}\left|\left\langle\left(\sum_{j=1}^{n} \alpha_{j} T_{j}\right) x, y\right\rangle\right|\right) \\
& =\sup _{\|\alpha\|_{n, p} \leq 1}\left(\sup _{\|y\|=1}\left|\left\langle\left(\sum_{j=1}^{n} \alpha_{j} T_{j}\right) x, y\right\rangle\right|\right) \\
& =\sup _{\|\alpha\|_{n, p} \leq 1}\left\|\left(\sum_{j=1}^{n} \alpha_{j} T_{j}\right) x\right\|,
\end{aligned}
$$

which proves the equality $(2.15)$.

The other equalities can be proved in a similar way by using Theorem 2 , however the details are omitted.

We can state and prove our main result.

Theorem 5. Let $\left(T_{1}, \ldots, T_{n}\right) \in B^{(n)}(H)$.

(i) For $q \geq 1$ we have the representation for the hypo-q-norm

$$
\begin{aligned}
\left\|\left(T_{1}, \ldots, T_{n}\right)\right\|_{h, n, q} & =\sup _{\|x\|=\|y\|=1}\left(\sum_{j=1}^{n}\left|\left\langle T_{j} x, y\right\rangle\right|^{q}\right)^{1 / q} \\
& =\left\|\left(T_{1}, \ldots, T_{n}\right)\right\|_{s, q}
\end{aligned}
$$

and in particular

$$
\left\|\left(T_{1}, \ldots, T_{n}\right)\right\|_{n, e}=\sup _{\|x\|=\|y\|=1}\left(\sum_{j=1}^{n}\left|\left\langle T_{j} x, y\right\rangle\right|^{2}\right)^{1 / 2} .
$$

We also have

$$
\left\|\left(T_{1}, \ldots, T_{n}\right)\right\|_{h, n, \infty}=\max _{j \in\{1, \ldots, n\}}\left\{\left\|T_{j}\right\|\right\} .
$$


(ii) For $q \geq 1$ we have the representation for the hypo--numerical radius

$$
\begin{aligned}
w_{h, n, q}\left(T_{1}, \ldots, T_{n}\right) & =\sup _{\|x\|=1}\left(\sum_{j=1}^{n}\left|\left\langle T_{j} x, x\right\rangle\right|^{q}\right)^{1 / q} \\
& =w_{s, q}\left(T_{1}, \ldots, T_{n}\right)
\end{aligned}
$$

and in particular

$$
w_{n, e}\left(T_{1}, \ldots, T_{n}\right):=\sup _{\|x\|=1}\left(\sum_{j=1}^{n}\left|\left\langle T_{j} x, x\right\rangle\right|^{2}\right)^{1 / 2} .
$$

We also have

$$
w_{h, n, \infty}\left(T_{1}, \ldots, T_{n}\right)=\max _{j \in\{1, \ldots, n\}}\left\{w\left(T_{j}\right)\right\}
$$

Proof. (i) By using the equality 2.15) we have for $\left(T_{1}, \ldots, T_{n}\right) \in B^{(n)}(H)$ that

$$
\begin{aligned}
\sup _{\|x\|=\|y\|=1}\left(\sum_{j=1}^{n}\left|\left\langle T_{j} x, y\right\rangle\right|^{q}\right)^{1 / q} & =\sup _{\|x\|=1}\left(\sup _{\|y\|=1}\left(\sum_{j=1}^{n}\left|\left\langle T_{j} x, y\right\rangle\right|^{q}\right)^{1 / q}\right) \\
& =\sup _{\|x\|=1}\left(\sup _{\|\alpha\|_{n, p} \leq 1}\left\|\sum_{j=1}^{n} \alpha_{j} T_{j} x\right\|\right) \\
& =\sup _{\|\alpha\|_{n, p} \leq 1}\left(\sup _{\|x\|=1}\left\|\sum_{j=1}^{n} \alpha_{j} T_{j} x\right\|\right) \\
& =\sup _{\|\alpha\|_{n, p} \leq 1}\left\|\sum_{j=1}^{n} \alpha_{j} T_{j}\right\| \\
& =\left\|\left(T_{1}, \ldots, T_{n}\right)\right\|_{h, n, q}
\end{aligned}
$$

which proves 2.19). The rest is obvious. 
(ii) By using the equality (2.11) we have for $\left(T_{1}, \ldots, T_{n}\right) \in B^{(n)}(H)$ that

$$
\begin{aligned}
\sup _{\|x\|=1}\left(\sum_{j=1}^{n}\left|\left\langle T_{j} x, x\right\rangle\right|^{q}\right)^{1 / q} & =\sup _{\|x\|=1}\left(\sup _{\|\alpha\|_{n, p} \leq 1} \mid\left\langle\left(\sum_{j=1}^{n} \alpha_{j} T_{j}\right) x, x\right\rangle\right) \\
& =\sup _{\|\alpha\|_{n, p} \leq 1}\left(\sup _{\|x\|=1} \mid\left\langle\left(\sum_{j=1}^{n} \alpha_{j} T_{j}\right) x, x\right\rangle\right) \\
& =\sup _{\|\alpha\|_{n, p} \leq 1} w\left(\sum_{j=1}^{n} \alpha_{j} T_{j}\right)=w_{h, n, q}\left(T_{1}, \ldots, T_{n}\right)
\end{aligned}
$$

which proves 2.22. The rest is obvious.

Remark 6 . The case $q=2$ was obtained in a different manner in [5] by utilising the rotation-invariant normalised positive Borel measure on the unit sphere.

We can consider on $B^{(n)}(H)$ the following usual operator and numerical radius $q$-norms, for $q \geq 1$

$$
\begin{aligned}
\left\|\left(T_{1}, \ldots, T_{n}\right)\right\|_{n, q} & :=\left(\sum_{j=1}^{n}\left\|T_{j}\right\|^{q}\right)^{1 / q}, \\
w_{n, q}\left(T_{1}, \ldots, T_{n}\right) & :=\left(\sum_{j=1}^{n} w^{q}\left(T_{j}\right)\right)^{1 / q},
\end{aligned}
$$

where $\left(T_{1}, \ldots, T_{n}\right) \in B^{(n)}(H)$. For $q=\infty$ we put

$$
\begin{aligned}
\left\|\left(T_{1}, \ldots, T_{n}\right)\right\|_{n, \infty} & :=\max _{j \in\{1, \ldots, n\}}\left\{\left\|T_{j}\right\|\right\}, \\
w_{n, \infty}\left(T_{1}, \ldots, T_{n}\right) & :=\max _{j \in\{1, \ldots, n\}}\left\{w\left(T_{j}\right)\right\} .
\end{aligned}
$$

COROLlary 7. With the assumptions of Theorem 5 we have for $q \geq 1$ that

$$
\frac{1}{n^{1 / q}}\left\|\left(T_{1}, \ldots, T_{n}\right)\right\|_{n, q} \leq\left\|\left(T_{1}, \ldots, T_{n}\right)\right\|_{h, n, q} \leq\left\|\left(T_{1}, \ldots, T_{n}\right)\right\|_{n, q}
$$


and

$$
\frac{1}{n^{1 / q}} w_{n, q}\left(T_{1}, \ldots, T_{n}\right) \leq w_{h, n, q}\left(T_{1}, \ldots, T_{n}\right) \leq w_{n, q}\left(T_{1}, \ldots, T_{n}\right)
$$

for any $\left(T_{1}, \ldots, T_{n}\right) \in B^{(n)}(H)$.

In particular, we have [5]

$$
\frac{1}{\sqrt{n}}\left\|\left(T_{1}, \ldots, T_{n}\right)\right\|_{n, 2} \leq\left\|\left(T_{1}, \ldots, T_{n}\right)\right\|_{h, n, e} \leq\left\|\left(T_{1}, \ldots, T_{n}\right)\right\|_{n, 2}
$$

and

$$
\frac{1}{\sqrt{n}} w_{n, 2}\left(T_{1}, \ldots, T_{n}\right) \leq w_{h, n, e}\left(T_{1}, \ldots, T_{n}\right) \leq w_{n, 2}\left(T_{1}, \ldots, T_{n}\right)
$$

for any $\left(T_{1}, \ldots, T_{n}\right) \in B^{(n)}(H)$.

Proof. Let $\left(T_{1}, \ldots, T_{n}\right) \in B^{(n)}(H)$ and $x, y \in H$ with $\|x\|=\|y\|=1$. Then by Schwarz's inequality we have

$$
\left(\sum_{j=1}^{n}\left|\left\langle T_{j} x, y\right\rangle\right|^{q}\right)^{1 / q} \leq\left(\sum_{j=1}^{n}\left\|T_{j} x\right\|^{q}\|y\|^{q}\right)^{1 / q}=\left(\sum_{j=1}^{n}\left\|T_{j} x\right\|^{q}\right)^{1 / q} .
$$

By the operator norm inequality we also have

$$
\left(\sum_{j=1}^{n}\left\|T_{j} x\right\|^{q}\right)^{1 / q} \leq\left(\sum_{j=1}^{n}\left\|T_{j}\right\|^{q}\|x\|^{q}\right)^{1 / q}=\left\|\left(T_{1}, \ldots, T_{n}\right)\right\|_{n, q} .
$$

Therefore

$$
\left(\sum_{j=1}^{n}\left|\left\langle T_{j} x, y\right\rangle\right|^{q}\right)^{1 / q} \leq\left\|\left(T_{1}, \ldots, T_{n}\right)\right\|_{n, q}
$$

and by taking the supremum over $\|x\|=\|y\|=1$ we get the second inequality in 2.25).

By the properties of complex numbers, we have

$$
\max _{j \in\{1, \ldots, n\}}\left\{\left|\left\langle T_{j} x, y\right\rangle\right|\right\} \leq\left(\sum_{j=1}^{n}\left|\left\langle T_{j} x, y\right\rangle\right|^{q}\right)^{1 / q}
$$

$x, y \in H$ with $\|x\|=\|y\|=1$. 
By taking the supremum over $\|x\|=\|y\|=1$ we get

$$
\sup _{\|x\|=\|y\|=1}\left(\max _{j \in\{1, \ldots, n\}}\left\{\left|\left\langle T_{j} x, y\right\rangle\right|\right\}\right) \leq\left\|\left(T_{1}, \ldots, T_{n}\right)\right\|_{h, n, q}
$$

and since

$$
\begin{aligned}
\sup _{\|x\|=\|y\|=1}\left(\max _{j \in\{1, \ldots, n\}}\left\{\left|\left\langle T_{j} x, y\right\rangle\right|\right\}\right) & =\max _{j \in\{1, \ldots, n\}}\left\{\sup _{\|x\|=\|y\|=1}\left|\left\langle T_{j} x, y\right\rangle\right|\right\} \\
& =\max _{j \in\{1, \ldots, n\}}\left\{\left\|T_{j}\right\|\right\}=\left\|\left(T_{1}, \ldots, T_{n} t\right)\right\|_{n, \infty}
\end{aligned}
$$

then by 2.29 we get

$$
\left\|\left(T_{1}, \ldots, T_{n}\right)\right\|_{n, \infty} \leq\left\|\left(T_{1}, \ldots, T_{n}\right)\right\|_{h, n, q}
$$

for any $\left(T_{1}, \ldots, T_{n}\right) \in B^{(n)}(H)$.

Since

$$
\begin{aligned}
\left\|\left(T_{1}, \ldots, T_{n}\right)\right\|_{n, q} & :=\left(\sum_{j=1}^{n}\left\|T_{j}\right\|^{q}\right)^{1 / q} \leq\left(n\left\|\left(T_{1}, \ldots, T_{n}\right)\right\|_{n, \infty}^{q}\right)^{1 / q} \\
& =n^{1 / q}\left\|\left(T_{1}, \ldots, T_{n}\right)\right\|_{n, \infty},
\end{aligned}
$$

then by 2.30 and 2.31 we get

$$
\frac{1}{n^{1 / q}}\left\|\left(T_{1}, \ldots, T_{n}\right)\right\|_{n, q} \leq\left\|\left(T_{1}, \ldots, T_{n}\right)\right\|_{h, n, q}
$$

for any $\left(T_{1}, \ldots, T_{n}\right) \in B^{(n)}(H)$.

The inequality (2.26) follows in a similar way and we omit the details.

Corollary 8. With the assumptions of Theorem 5 we have for $r \geq q \geq 1$ that

$$
\left\|\left(T_{1}, \ldots, T_{n}\right)\right\|_{h, n, r} \leq\left\|\left(T_{1}, \ldots, T_{n}\right)\right\|_{h, n, q} \leq n^{\frac{r-q}{r q}}\left\|\left(T_{1}, \ldots, T_{n}\right)\right\|_{h, n, r}
$$

and 14

$$
w_{h, n, r}\left(T_{1}, \ldots, T_{n}\right) \leq w_{h, n, q}\left(T_{1}, \ldots, T_{n}\right) \leq n^{\frac{r-q}{r q}} w_{h, n, r}\left(T_{1}, \ldots, T_{n}\right)
$$

for any $\left(T_{1}, \ldots, T_{n}\right) \in B^{(n)}(H)$. 
Proof. We use the following elementary inequalities for the nonnegative numbers $a_{j}, j=1, \ldots, n$ and $r \geq q>0$ (see for instance [14])

$$
\left(\sum_{j=1}^{n} a_{j}^{r}\right)^{1 / r} \leq\left(\sum_{j=1}^{n} a_{j}^{q}\right)^{1 / q} \leq n^{\frac{r-q}{r q}}\left(\sum_{j=1}^{n} a_{j}^{r}\right)^{1 / r}
$$

Let $\left(T_{1}, \ldots, T_{n}\right) \in B^{(n)}(H)$ and $x, y \in H$ with $\|x\|=\|y\|=1$. Then by (2.34) we get

$$
\left(\sum_{j=1}^{n}\left|\left\langle T_{j} x, y\right\rangle\right|^{r}\right)^{1 / r} \leq\left(\sum_{j=1}^{n}\left|\left\langle T_{j} x, y\right\rangle\right|^{q}\right)^{1 / q} \leq n^{\frac{r-q}{r q}}\left(\sum_{j=1}^{n}\left|\left\langle T_{j} x, y\right\rangle\right|^{r}\right)^{1 / r} .
$$

By taking the supremum over $\|x\|=\|y\|=1$ we get (2.32).

The inequality (2.33) follows in a similar way and we omit the details.

Remark 9. For $q \geq 2$ we have by 2.32 and 2.33

$$
\left\|\left(T_{1}, \ldots, T_{n}\right)\right\|_{h, n, q} \leq\left\|\left(T_{1}, \ldots, T_{n}\right)\right\|_{h, n, e} \leq n^{\frac{q-2}{2 q}}\left\|\left(T_{1}, \ldots, T_{n}\right)\right\|_{h, n, q}
$$

and

$$
w_{h, n, q}\left(T_{1}, \ldots, T_{n}\right) \leq w_{h, n, e}\left(T_{1}, \ldots, T_{n}\right) \leq n^{\frac{q-2}{2 q}} w_{h, n, q}\left(T_{1}, \ldots, T_{n}\right)
$$

and for $1 \leq q \leq 2$ we have

$$
\left\|\left(T_{1}, \ldots, T_{n}\right)\right\|_{h, n, e} \leq\left\|\left(T_{1}, \ldots, T_{n}\right)\right\|_{h, n, q} \leq n^{\frac{2-q}{2 q}}\left\|\left(T_{1}, \ldots, T_{n}\right)\right\|_{h, n, e}
$$

and

$$
w_{h, n, e}\left(T_{1}, \ldots, T_{n}\right) \leq w_{h, n, e}\left(T_{1}, \ldots, T_{n}\right) \leq n^{\frac{2-q}{2 q}} w_{h, n, e}\left(T_{1}, \ldots, T_{n}\right)
$$

for any $\left(T_{1}, \ldots, T_{n}\right) \in B^{(n)}(H)$.

Also, if we take $q=1$ and $r \geq 1$ in 2.32 and (2.33), then we get

$$
\left\|\left(T_{1}, \ldots, T_{n}\right)\right\|_{h, n, r} \leq\left\|\left(T_{1}, \ldots, T_{n}\right)\right\|_{h, n, 1} \leq n^{\frac{r-1}{r}}\left\|\left(T_{1}, \ldots, T_{n}\right)\right\|_{h, n, r}
$$

and

$$
w_{h, n, r}\left(T_{1}, \ldots, T_{n}\right) \leq w_{h, n, 1}\left(T_{1}, \ldots, T_{n}\right) \leq n^{\frac{r-1}{r}} w_{h, n, r}\left(T_{1}, \ldots, T_{n}\right)
$$

for any $\left(T_{1}, \ldots, T_{n}\right) \in B^{(n)}(H)$. 
In particular, for $r=2$ we get

$$
\left\|\left(T_{1}, \ldots, T_{n}\right)\right\|_{h, n, e} \leq\left\|\left(T_{1}, \ldots, T_{n}\right)\right\|_{h, n, 1} \leq \sqrt{n}\left\|\left(T_{1}, \ldots, T_{n}\right)\right\|_{h, n, e}
$$

and

$$
w_{n, e}\left(T_{1}, \ldots, T_{n}\right) \leq w_{h, n, 1}\left(T_{1}, \ldots, T_{n}\right) \leq \sqrt{n} w_{n, e}\left(T_{1}, \ldots, T_{n}\right)
$$

for any $\left(T_{1}, \ldots, T_{n}\right) \in B^{(n)}(H)$.

We have:

Proposition 10. For any $\left(T_{1}, \ldots, T_{n}\right) \in B^{(n)}(H)$ and $p, q>1$ with $\frac{1}{p}+\frac{1}{q}=1$, then we have

$$
\left\|\left(T_{1}, \ldots, T_{n}\right)\right\|_{h, n, q} \geq \frac{1}{n^{1 / p}}\left\|\sum_{j=1}^{n} T_{j}\right\|
$$

and

$$
w_{h, n, q}\left(T_{1}, \ldots, T_{n}\right) \geq \frac{1}{n^{1 / p}} w\left(\sum_{j=1}^{n} T_{j}\right)
$$

Proof. Let $\lambda_{j}=\frac{1}{n^{1 / p}}$ for $j \in\{1, \ldots, n\}$, then $\sum_{j=1}^{n}\left|\lambda_{j}\right|^{p}=1$. Therefore by 1.8 we get

$$
\left\|\left(T_{1}, \ldots, T_{n}\right)\right\|_{h, n, q}=\sup _{\|\lambda\|_{n, p} \leq 1}\left\|\sum_{j=1}^{n} \lambda_{j} T_{j}\right\| \geq\left\|\sum_{j=1}^{n} \frac{1}{n^{1 / p}} T_{j}\right\|=\frac{1}{n^{1 / p}}\left\|\sum_{j=1}^{n} T_{j}\right\| .
$$

The inequality (2.44) follows in a similar way.

We can also introduce the following norms for $\left(T_{1}, \ldots, T_{n}\right) \in B^{(n)}(H)$,

$$
\left\|\left(T_{1}, \ldots, T_{n}\right)\right\|_{s, n, p}:=\sup _{\|x\|=1}\left(\sum_{j=1}^{n}\left\|T_{j} x\right\|^{p}\right)^{1 / p}
$$

where $p \geq 1$ and

$$
\left\|\left(T_{1}, \ldots, T_{n}\right)\right\|_{s, n, \infty}:=\sup _{\|x\|=1}\left(\max _{j \in\{1, \ldots, n\}}\left\|T_{j} x\right\|\right)=\max _{j \in\{1, \ldots, n\}}\left\{\left\|T_{j}\right\|\right\} .
$$

The triangle inequality $\|\cdot\|_{s, n, q}$ follows by Minkowski inequality, while the other properties of the norm are obvious. 
Proposition 11. Let $\left(T_{1}, \ldots, T_{n}\right) \in B^{(n)}(H)$.

(i) We have for $p \geq 1$, that

$$
\begin{aligned}
\left\|\left(T_{1}, \ldots, T_{n}\right)\right\|_{h, n, p} & \leq\left\|\left(T_{1}, \ldots, T_{n}\right)\right\|_{s, n, p} \\
& \leq\left\|\left(T_{1}, \ldots, T_{n}\right)\right\|_{n, p},
\end{aligned}
$$

(ii) For $p \geq 2$ we also have

$$
\left\|\left(T_{1}, \ldots, T_{n}\right)\right\|_{s, n, p}=\left[w_{h, n, p / 2}\left(\left|T_{1}\right|^{2}, \ldots,\left|T_{n}\right|^{2}\right)\right]^{1 / 2}
$$

where the absolute value $|T|$ is defined by $|T|:=\left(T^{*} T\right)^{1 / 2}$.

Proof. (i) We have for $p \geq 2$ and $x, y \in H$ with $\|x\|=\|y\|=1$, that

$$
\begin{aligned}
\left|\left\langle T_{j} x, y\right\rangle\right|^{p} & \leq\left\|T_{j} x\right\|^{p}\|y\|^{p} \\
& =\left\|T_{j} x\right\|^{p} \leq\left\|T_{j}\right\|^{p}\|x\|^{p}=\left\|T_{j}\right\|^{p}
\end{aligned}
$$

for $j \in\{1, \ldots, n\}$.

This implies

$$
\sum_{j=1}^{n}\left|\left\langle T_{j} x, y\right\rangle\right|^{p} \leq \sum_{j=1}^{n}\left\|T_{j} x\right\|^{p} \leq \sum_{j=1}^{n}\left\|T_{j}\right\|^{p},
$$

namely

$$
\left(\sum_{j=1}^{n}\left|\left\langle T_{j} x, y\right\rangle\right|^{p}\right)^{1 / p} \leq\left(\sum_{j=1}^{n}\left\|T_{j} x\right\|^{p}\right)^{1 / p} \leq\left(\sum_{j=1}^{n}\left\|T_{j}\right\|^{p}\right)^{1 / p},
$$

for any $x, y \in H$ with $\|x\|=\|y\|=1$.

Taking the supremum over $\|x\|=\|y\|=1$ in (2.47), we get the desired result (2.45). 
(ii) We have

$$
\begin{aligned}
& \left\|\left(T_{1}, \ldots, T_{n}\right)\right\|_{s, n, p} \\
& =\sup _{\|x\|=1}\left(\sum_{j=1}^{n}\left\|T_{j} x\right\|^{p}\right)^{1 / p}=\sup _{\|x\|=1}\left(\sum_{j=1}^{n}\left(\left\|T_{j} x\right\|^{2}\right)^{p / 2}\right)^{1 / p} \\
& =\sup _{\|x\|=1}\left(\sum_{j=1}^{n}\left\langle T_{j} x, T_{j} x\right\rangle^{p / 2}\right)^{1 / p}=\sup _{\|x\|=1}\left(\sum_{j=1}^{n}\left\langle T_{j}^{*} T_{j} x, x\right\rangle^{p / 2}\right)^{1 / p} \\
& =\sup _{\|x\|=1}\left(\sum_{j=1}^{n}\left\langle\left|T_{j}\right|^{2} x, x\right\rangle^{p / 2}\right)^{1 / p}=\left[\sup _{\|x\|=1}\left(\sum_{j=1}^{n}\left\langle\left|T_{j}\right|^{2} x, x\right\rangle^{p / 2}\right)^{1 /(p / 2)}\right]^{1 / 2} \\
& =\left[w_{h, n, p / 2}\left(\left|T_{1}\right|^{2}, \ldots,\left|T_{n}\right|^{2}\right)\right]^{1 / 2},
\end{aligned}
$$

which proves the equality 2.46 .

\section{Some REVERSE INEQUALITIES}

Recall the following reverse of Cauchy-Buniakowski-Schwarz inequality [2] (see also [3, Theorem 5.14]):

Lemma 12. Let $a, A \in \mathbb{R}$ and $\mathbf{z}=\left(z_{1}, \ldots, z_{n}\right), \mathbf{y}=\left(y_{1}, \ldots, y_{n}\right)$ be two sequences of real numbers with the property that:

$$
a y_{j} \leq z_{j} \leq A y_{j} \quad \text { for each } j \in\{1, \ldots, n\} .
$$

Then for any $\mathbf{w}=\left(w_{1}, \ldots, w_{n}\right)$ a sequence of positive real numbers, one has the inequality

$$
0 \leq \sum_{j=1}^{n} w_{j} z_{j}^{2} \sum_{j=1}^{n} w_{j} y_{j}^{2}-\left(\sum_{j=1}^{n} w_{j} z_{j} y_{j}\right)^{2} \leq \frac{1}{4}(A-a)^{2}\left(\sum_{j=1}^{n} w_{j} y_{j}^{2}\right)^{2} .
$$

The constant $\frac{1}{4}$ is sharp in 3.2 .

O. Shisha and B. Mond obtained in 1967 (see [15]) the following counterparts of $(C B S)$-inequality (see also [3, Theorem $5.20 \& 5.21]$ ): 
Lemma 13. Assume that $\mathbf{a}=\left(a_{1}, \ldots, a_{n}\right)$ and $\mathbf{b}=\left(b_{1}, \ldots, b_{n}\right)$ are such that there exists $a, A, b, B$ with the property that:

$$
0 \leq a \leq a_{j} \leq A \quad \text { and } \quad 0<b \leq b_{j} \leq B \quad \text { for any } j \in\{1, \ldots, n\},
$$

then we have the inequality

$$
\sum_{j=1}^{n} a_{j}^{2} \sum_{j=1}^{n} b_{j}^{2}-\left(\sum_{j=1}^{n} a_{j} b_{j}\right)^{2} \leq\left(\sqrt{\frac{A}{b}}-\sqrt{\frac{a}{B}}\right)^{2} \sum_{j=1}^{n} a_{j} b_{j} \sum_{j=1}^{n} b_{j}^{2} .
$$

and

Lemma 14. Assume that $\mathbf{a}, \mathbf{b}$ are nonnegative sequences and there exists $\gamma, \Gamma$ with the property that

$$
0 \leq \gamma \leq \frac{a_{j}}{b_{j}} \leq \Gamma<\infty \quad \text { for any } j \in\{1, \ldots, n\} .
$$

Then we have the inequality

$$
0 \leq\left(\sum_{j=1}^{n} a_{j}^{2} \sum_{j=1}^{n} b_{j}^{2}\right)^{\frac{1}{2}}-\sum_{j=1}^{n} a_{j} b_{j} \leq \frac{(\Gamma-\gamma)^{2}}{4(\gamma+\Gamma)} \sum_{j=1}^{n} b_{j}^{2} .
$$

We have:

TheOREM 15. Let $\left(T_{1}, \ldots, T_{n}\right) \in B^{(n)}(H)$.

(i) We have

$$
\begin{aligned}
0 & \leq\left\|\left(T_{1}, \ldots, T_{n}\right)\right\|_{h, n, e}^{2}-\frac{1}{n}\left\|\left(T_{1}, \ldots, T_{n}\right)\right\|_{h, n, 1}^{2} \\
& \leq \frac{1}{4} n\left\|\left(T_{1}, \ldots, T_{n}\right)\right\|_{n, \infty}^{2}
\end{aligned}
$$

and

$$
\begin{aligned}
0 & \leq w_{n, e}^{2}\left(T_{1}, \ldots, T_{n}\right)-\frac{1}{n} w_{h, n, 1}^{2}\left(T_{1}, \ldots, T_{n}\right) \\
& \leq \frac{1}{4} n\left\|\left(T_{1}, \ldots, T_{n}\right)\right\|_{n, \infty}^{2} .
\end{aligned}
$$


(ii) We have

$$
\begin{aligned}
0 & \leq\left\|\left(T_{1}, \ldots, T_{n}\right)\right\|_{h, n, e}^{2}-\frac{1}{n}\left\|\left(T_{1}, \ldots, T_{n}\right)\right\|_{h, n, 1}^{2} \\
& \leq\left\|\left(T_{1}, \ldots, T_{n}\right)\right\|_{n, \infty}\left\|\left(T_{1}, \ldots, T_{n}\right)\right\|_{h, n, 1}
\end{aligned}
$$

and

$$
\begin{aligned}
0 & \leq w_{n, e}^{2}\left(T_{1}, \ldots, T_{n}\right)-\frac{1}{n} w_{h, n, 1}^{2}\left(T_{1}, \ldots, T_{n}\right) \\
& \leq\left\|\left(T_{1}, \ldots, T_{n}\right)\right\|_{n, \infty} w_{h, n, 1}\left(T_{1}, \ldots, T_{n}\right)
\end{aligned}
$$

(iii) We have

$$
\begin{aligned}
0 & \leq\left\|\left(T_{1}, \ldots, T_{n}\right)\right\|_{h, n, e}-\frac{1}{\sqrt{n}}\left\|\left(T_{1}, \ldots, T_{n}\right)\right\|_{h, n, 1} \\
& \leq \frac{1}{4} \sqrt{n}\left\|\left(T_{1}, \ldots, T_{n}\right)\right\|_{n, \infty}
\end{aligned}
$$

and

$$
\begin{aligned}
0 & \leq w_{n, e}\left(T_{1}, \ldots, T_{n}\right)-\frac{1}{\sqrt{n}} w_{h, n, 1}\left(T_{1}, \ldots, T_{n}\right) \\
& \leq \frac{1}{4} \sqrt{n}\left\|\left(T_{1}, \ldots, T_{n}\right)\right\|_{n, \infty} .
\end{aligned}
$$

Proof. (i) Let $\left(T_{1}, \ldots, T_{n}\right) \in B^{(n)}(H)$ and put

$$
R=\max _{j \in\{1, \ldots, n\}}\left\{\left\|T_{j}\right\|\right\}=\left\|\left(T_{1}, \ldots, T_{n}\right)\right\|_{n, \infty} .
$$

If $x, y \in H$, with $\|x\|=\|y\|=1$ then $\left|\left\langle T_{j} x, y\right\rangle\right| \leq\left\|T_{j} x\right\| \leq\left\|T_{j}\right\| \leq R$ for any $j \in\{1, \ldots, n\}$.

If we write the inequality (3.2) for $z_{j}=\left|\left\langle T_{j} x, y\right\rangle\right|, w_{j}=y_{j}=1, A=R$ and $a=0$, we get

$$
0 \leq n \sum_{j=1}^{n}\left|\left\langle T_{j} x, y\right\rangle\right|^{2}-\left(\sum_{j=1}^{n}\left|\left\langle T_{j} x, y\right\rangle\right|\right)^{2} \leq \frac{1}{4} n^{2} R^{2}
$$

for any $x, y \in H$, with $\|x\|=\|y\|=1$. 
This implies that

$$
\sum_{j=1}^{n}\left|\left\langle T_{j} x, y\right\rangle\right|^{2} \leq \frac{1}{n}\left(\sum_{j=1}^{n}\left|\left\langle T_{j} x, y\right\rangle\right|\right)^{2}+\frac{1}{4} n R^{2}
$$

for any $x, y \in H$, with $\|x\|=\|y\|=1$ and, in particular

$$
\sum_{j=1}^{n}\left|\left\langle T_{j} x, x\right\rangle\right|^{2} \leq \frac{1}{n}\left(\sum_{j=1}^{n}\left|\left\langle T_{j} x, x\right\rangle\right|\right)^{2}+\frac{1}{4} n R^{2}
$$

for any $x \in H$, with $\|x\|=1$.

Taking the supremum over $\|x\|=\|y\|=1$ in (3.13) and $\|x\|=1$ in (3.14), then we get (3.7) and (3.8).

(ii) Let $\left(T_{1}, \ldots, T_{n}\right) \in B^{(n)}(H)$. If we write the inequality (3.4) for $a_{j}=$ $\left|\left\langle T_{j} x, y\right\rangle\right|, b_{j}=1, b=B=1, a=0$ and $A=R$, then we get

$$
0 \leq n \sum_{j=1}^{n}\left|\left\langle T_{j} x, y\right\rangle\right|^{2}-\left(\sum_{j=1}^{n}\left|\left\langle T_{j} x, y\right\rangle\right|\right)^{2} \leq n R \sum_{j=1}^{n}\left|\left\langle T_{j} x, y\right\rangle\right|,
$$

for any $x, y \in H$, with $\|x\|=\|y\|=1$.

This implies that

$$
\sum_{j=1}^{n}\left|\left\langle T_{j} x, y\right\rangle\right|^{2} \leq \frac{1}{n}\left(\sum_{j=1}^{n}\left|\left\langle T_{j} x, y\right\rangle\right|\right)^{2}+R \sum_{j=1}^{n}\left|\left\langle T_{j} x, y\right\rangle\right|,
$$

for any $x, y \in H$, with $\|x\|=\|y\|=1$ and, in particular

$$
\sum_{j=1}^{n}\left|\left\langle T_{j} x, x\right\rangle\right|^{2} \leq \frac{1}{n}\left(\sum_{j=1}^{n}\left|\left\langle T_{j} x, x\right\rangle\right|\right)^{2}+R \sum_{j=1}^{n}\left|\left\langle T_{j} x, x\right\rangle\right|,
$$

for any $x \in H$ with $\|x\|=1$.

Taking the supremum over $\|x\|=\|y\|=1$ in (3.15) and $\|x\|=1$ in (3.16), then we get $(3.9)$ and $(3.10)$.

(iii) If we write the inequality (3.6) for $a_{j}=\left|\left\langle T_{j} x, y\right\rangle\right|, b_{j}=1, b=B=1$, $\gamma=0$ and $\Gamma=R$ we have

$$
0 \leq\left(n \sum_{j=1}^{n}\left|\left\langle T_{j} x, y\right\rangle\right|^{2}\right)^{\frac{1}{2}}-\sum_{j=1}^{n}\left|\left\langle T_{j} x, y\right\rangle\right| \leq \frac{1}{4} n R,
$$


for any $x, y \in H$, with $\|x\|=\|y\|=1$.

This implies that

$$
\left(\sum_{j=1}^{n}\left|\left\langle T_{j} x, y\right\rangle\right|^{2}\right)^{\frac{1}{2}} \leq \frac{1}{\sqrt{n}} \sum_{j=1}^{n}\left|\left\langle T_{j} x, y\right\rangle\right|+\frac{1}{4} \sqrt{n} R,
$$

for any $x, y \in H$, with $\|x\|=\|y\|=1$ and, in particular

$$
\left(\sum_{j=1}^{n}\left|\left\langle T_{j} x, x\right\rangle\right|^{2}\right)^{\frac{1}{2}} \leq \frac{1}{\sqrt{n}} \sum_{j=1}^{n}\left|\left\langle T_{j} x, x\right\rangle\right|+\frac{1}{4} \sqrt{n} R,
$$

for any $x \in H$ with $\|x\|=1$.

Taking the supremum over $\|x\|=\|y\|=1$ in (3.17) and $\|x\|=1$ in (3.18), then we get (3.11) and (3.12).

Before we proceed with establishing some reverse inequalities for the hypoEuclidean numerical radius, we recall some reverse results of the CauchyBunyakovsky-Schwarz inequality for complex numbers as follows:

If $\gamma, \Gamma \in \mathbb{C}$ and $\alpha_{j} \in \mathbb{C}, j \in\{1, \ldots, n\}$ with the property that

$$
\begin{aligned}
0 & \leq \operatorname{Re}\left[\left(\Gamma-\alpha_{j}\right)\left(\overline{\alpha_{j}}-\bar{\gamma}\right)\right] \\
& =\left(\operatorname{Re} \Gamma-\operatorname{Re} \alpha_{j}\right)\left(\operatorname{Re} \alpha_{j}-\operatorname{Re} \gamma\right)+\left(\operatorname{Im} \Gamma-\operatorname{Im} \alpha_{j}\right)\left(\operatorname{Im} \alpha_{j}-\operatorname{Im} \gamma\right)
\end{aligned}
$$

or, equivalently,

$$
\left|\alpha_{j}-\frac{\gamma+\Gamma}{2}\right| \leq \frac{1}{2}|\Gamma-\gamma|
$$

for each $j \in\{1, \ldots, n\}$, then (see for instance [4, p. 9])

$$
n \sum_{j=1}^{n}\left|\alpha_{j}\right|^{2}-\left|\sum_{j=1}^{n} \alpha_{j}\right|^{2} \leq \frac{1}{4} n^{2}|\Gamma-\gamma|^{2} .
$$

In addition, if $\operatorname{Re}(\Gamma \bar{\gamma})>0$, then (see for example [4, p. 26]):

$$
\begin{aligned}
n \sum_{j=1}^{n}\left|\alpha_{j}\right|^{2} & \leq \frac{1}{4} \frac{\left\{\operatorname{Re}\left[(\bar{\Gamma}+\bar{\gamma}) \sum_{j=1}^{n} \alpha_{j}\right]\right\}^{2}}{\operatorname{Re}}(\Gamma \bar{\gamma}) \\
& \leq \frac{1}{4} \frac{|\Gamma+\gamma|^{2}}{\operatorname{Re}}(\Gamma \bar{\gamma})\left|\sum_{j=1}^{n} \alpha_{j}\right|^{2} .
\end{aligned}
$$


Also, if $\Gamma \neq-\gamma$, then (see for instance [4, p. 32]):

$$
\left(n \sum_{j=1}^{n}\left|\alpha_{j}\right|^{2}\right)^{\frac{1}{2}}-\left|\sum_{j=1}^{n} \alpha_{j}\right| \leq \frac{1}{4} n \frac{|\Gamma-\gamma|^{2}}{|\Gamma+\gamma|} .
$$

Finally, from [7] we can also state that

$$
n \sum_{j=1}^{n}\left|\alpha_{j}\right|^{2}-\left|\sum_{j=1}^{n} \alpha_{j}\right|^{2} \leq n[|\Gamma+\gamma|-2 \sqrt{\operatorname{Re}(\Gamma \bar{\gamma})}]\left|\sum_{j=1}^{n} \alpha_{j}\right|,
$$

provided $\operatorname{Re}(\Gamma \bar{\gamma})>0$.

We notice that a simple sufficient condition for 3.19 to hold is that

$$
\operatorname{Re} \Gamma \geq \operatorname{Re} \alpha_{j} \geq \operatorname{Re} \gamma \quad \text { and } \quad \operatorname{Im} \Gamma \geq \operatorname{Im} \alpha_{j} \geq \operatorname{Im} \gamma
$$

for each $j \in\{1, \ldots, n\}$.

Theorem 16. Let $\left(T_{1}, \ldots, T_{n}\right) \in B^{(n)}(H)$ and $\gamma, \Gamma \in \mathbb{C}$ with $\Gamma \neq \gamma$. Assume that

$$
w\left(T_{j}-\frac{\gamma+\Gamma}{2} I\right) \leq \frac{1}{2}|\Gamma-\gamma| \quad \text { for any } j \in\{1, \ldots, n\} .
$$

(i) We have

$$
w_{h, n, e}^{2}\left(T_{1}, \ldots, T_{n}\right) \leq \frac{1}{n} w^{2}\left(\sum_{j=1}^{n} T_{j}\right)+\frac{1}{4} n|\Gamma-\gamma|^{2} .
$$

(ii) If $\operatorname{Re}(\Gamma \bar{\gamma})>0$, then

$$
w_{h, n, e}\left(T_{1}, \ldots, T_{n}\right) \leq \frac{1}{2 \sqrt{n}} \frac{|\Gamma+\gamma|}{\sqrt{(\Gamma \bar{\gamma})}} w\left(\sum_{j=1}^{n} T_{j}\right)
$$

and

$$
\begin{aligned}
w_{h, n, e}^{2}( & \left.T_{1}, \ldots, T_{n}\right) \\
\leq & {\left[\frac{1}{n} w\left(\sum_{j=1}^{n} T_{j}\right)+[|\Gamma+\gamma|-2 \sqrt{(\Gamma \bar{\gamma})}]\right] \cdot w\left(\sum_{j=1}^{n} T_{j}\right) }
\end{aligned}
$$


(iii) If $\Gamma \neq-\gamma$, then

$$
w_{h, n, e}\left(T_{1}, \ldots, T_{n}\right) \leq \frac{1}{\sqrt{n}}\left(w\left(\sum_{j=1}^{n} T_{j}\right)+\frac{1}{4} \frac{|\Gamma-\gamma|^{2}}{|\Gamma+\gamma|}\right) .
$$

Proof. Let $x \in H$ with $\|x\|=1$ and $\left(T_{1}, \ldots, T_{n}\right) \in B^{(n)}(H)$ with the property (3.26). By taking $\alpha_{j}=\left\langle T_{j} x, x\right\rangle$ we have

$$
\begin{aligned}
\left|\alpha_{j}-\frac{\gamma+\Gamma}{2}\right| & =\left|\left\langle T_{j} x, x\right\rangle-\frac{\gamma+\Gamma}{2}\langle x, x\rangle\right|=\left|\left\langle\left(T_{j}-\frac{\gamma+\Gamma}{2} I\right) x, x\right\rangle\right| \\
& \leq \sup _{\|x\|=1}\left|\left\langle\left(T_{j}-\frac{\gamma+\Gamma}{2} I\right) x, x\right\rangle\right| \\
& =w\left(T_{j}-\frac{\gamma+\Gamma}{2}\right) \leq \frac{1}{2}|\Gamma-\gamma|
\end{aligned}
$$

for any $j \in\{1, \ldots, n\}$.

(i) By using the inequality (3.21), we have

$$
\begin{aligned}
\sum_{j=1}^{n}\left|\left\langle T_{j} x, x\right\rangle\right|^{2} & \leq \frac{1}{n}\left|\sum_{j=1}^{n}\left\langle T_{j} x, x\right\rangle\right|^{2}+\frac{1}{4} n|\Gamma-\gamma|^{2} \\
& =\frac{1}{n}\left|\left\langle\sum_{j=1}^{n} T_{j} x, x\right\rangle\right|^{2}+\frac{1}{4} n|\Gamma-\gamma|^{2}
\end{aligned}
$$

for any $x \in H$ with $\|x\|=1$.

By taking the supremum over $\|x\|=1$ in (3.31) we get

$$
\begin{aligned}
\sup _{\|x\|=1}\left(\sum_{j=1}^{n}\left|\left\langle T_{j} x, x\right\rangle\right|^{2}\right) & \leq \frac{1}{n} \sup _{\|x\|=1}\left|\left\langle\sum_{j=1}^{n} T_{j} x, x\right\rangle\right|^{2}+\frac{1}{4} n|\Gamma-\gamma|^{2} \\
& =\frac{1}{n} w^{2}\left(\sum_{j=1}^{n} T_{j}\right)+\frac{1}{4} n|\Gamma-\gamma|^{2}
\end{aligned}
$$

which proves $(3.27)$. 
(ii) If $\operatorname{Re}(\Gamma \bar{\gamma})>0$, then by 3.22 we have for $\alpha_{j}=\left\langle T_{j} x, x\right\rangle, j \in\{1, \ldots, n\}$ that

$$
\begin{aligned}
\sum_{j=1}^{n}\left|\left\langle T_{j} x, x\right\rangle\right|^{2} & \leq \frac{1}{4 n} \frac{|\Gamma+\gamma|^{2}}{\operatorname{Re}(\Gamma \bar{\gamma})}\left|\sum_{j=1}^{n}\left\langle T_{j} x, x\right\rangle\right|^{2} \\
& =\frac{1}{4 n} \frac{|\Gamma+\gamma|^{2}}{\operatorname{Re}(\Gamma \bar{\gamma})}\left|\left\langle\sum_{j=1}^{n} T_{j} x, x\right\rangle\right|^{2}
\end{aligned}
$$

for any $x \in H$ with $\|x\|=1$.

On taking the supremum over $\|x\|=1$ in 3.32 ) we get (3.32).

Also, by (3.24) we get

$$
\sum_{j=1}^{n}\left|\left\langle T_{j} x, x\right\rangle\right|^{2} \leq \frac{1}{n}\left|\sum_{j=1}^{n}\left\langle T_{j} x, x\right\rangle\right|^{2}+[|\Gamma+\gamma|-2 \sqrt{\operatorname{Re}(\Gamma \bar{\gamma})}]\left|\sum_{j=1}^{n}\left\langle T_{j} x, x\right\rangle\right|
$$

for any $x \in H$ with $\|x\|=1$.

By taking the supremum over $\|x\|=1$ in this inequality, we have

$$
\begin{aligned}
\sup _{\|x\|=1} & \sum_{j=1}^{n}\left|\left\langle T_{j} x, x\right\rangle\right|^{2} \\
& \leq \sup _{\|x\|=1}\left[\frac{1}{n}\left|\sum_{j=1}^{n}\left\langle T_{j} x, x\right\rangle\right|^{2}+[|\Gamma+\gamma|-2 \sqrt{(\Gamma \bar{\gamma})}]\left|\sum_{j=1}^{n}\left\langle T_{j} x, x\right\rangle\right|\right] \\
& \leq \frac{1}{n} \sup _{\|x\|=1}\left|\left\langle\sum_{j=1}^{n} T_{j} x, x\right\rangle\right|^{2}+[|\Gamma+\gamma|-2 \sqrt{(\Gamma \bar{\gamma})}] \sup _{\|x\|=1}\left|\left\langle\sum_{j=1}^{n} T_{j} x, x\right\rangle\right| \\
& =\frac{1}{n} w^{2}\left(\sum_{j=1}^{n} T_{j}\right)+[|\Gamma+\gamma|-2 \sqrt{(\Gamma \bar{\gamma})}] w\left(\sum_{j=1}^{n} T_{j}\right),
\end{aligned}
$$

which proves $(3.29)$. 
(iii) By the inequality $(3.23)$ we have

$$
\begin{aligned}
\left(\sum_{j=1}^{n}\left|\left\langle T_{j} x, x\right\rangle\right|^{2}\right)^{\frac{1}{2}} & \leq \frac{1}{\sqrt{n}}\left(\left|\sum_{j=1}^{n}\left\langle T_{j} x, x\right\rangle\right|+\frac{1}{4} \frac{|\Gamma-\gamma|^{2}}{|\Gamma+\gamma|}\right) \\
& =\frac{1}{\sqrt{n}}\left(\left|\left\langle\sum_{j=1}^{n} T_{j} x, x\right\rangle\right|+\frac{1}{4} \frac{|\Gamma-\gamma|^{2}}{|\Gamma+\gamma|}\right)
\end{aligned}
$$

for any $x \in H$ with $\|x\|=1$.

By taking the supremum over $\|x\|=1$ in this inequality, we get (3.30).

Remark 17. By the use of the elementary inequality $w(T) \leq\|T\|$ that holds for any $T \in B(H)$, a sufficient condition for $(3.26)$ to hold is that

$$
\left\|T_{j}-\frac{\gamma+\Gamma}{2}\right\| \leq \frac{1}{2}|\Gamma-\gamma| \quad \text { for any } j \in\{1, \ldots, n\} .
$$

4. INEQUALITIES FOR $\delta_{p}$ AND $\vartheta_{p}$ NORMS

For $T \in B(H)$ and $p \geq 1$ we can consider the functionals

$$
\delta_{p}(T):=\sup _{\|x\|=\|y\|=1}\left(|\langle T x, y\rangle|^{p}+\left|\left\langle T^{*} x, y\right\rangle\right|^{p}\right)^{1 / p}=\left\|\left(T, T^{*}\right)\right\|_{h, 2, p}
$$

and

$$
\vartheta_{p}(T):=\sup _{\|x\|=1}\left(\|T x\|^{p}+\left\|T^{*} x\right\|^{p}\right)^{1 / p}=\left\|\left(T, T^{*}\right)\right\|_{s, 2, p} .
$$

It is easy to see that both $\delta_{p}$ and $\vartheta_{p}$ are norms on $B(H)$. The case $p=2$ for the norm $\delta:=\delta_{2}$ was considered and studied in [5].

Observe that, for any $T \in B(H)$ and $p \geq 1$, we have

$$
\begin{aligned}
w_{h, 2, p}\left(\left(T, T^{*}\right)\right) & =\sup _{\|x\|=1}\left(|\langle T x, x\rangle|^{p}+\left|\left\langle T^{*} x, x\right\rangle\right|^{p}\right)^{1 / p} \\
& =\sup _{\|x\|=1}\left(|\langle T x, x\rangle|^{p}+|\langle T x, x\rangle|^{p}\right)^{1 / p} \\
& =2^{1 / p} \sup _{\|x\|=1}|\langle T x, x\rangle|=2^{1 / p} w(T) .
\end{aligned}
$$

Using the inequality (1.13) we have

$$
2^{1 / p} w(T) \leq \delta_{p}(T) \leq 2^{1+1 / p} w(T)
$$


for any $T \in B(H)$ and $p \geq 1$.

For $p=2$, we get

$$
\sqrt{2} w(T) \leq \delta(T) \leq \sqrt{8} w(T)
$$

while for $p=1$ we get

$$
2 w(T) \leq \delta_{1}(T) \leq 4 w(T)
$$

for any $T \in B(H)$.

We have for any $T \in B(H)$ and $p \geq 1$ that

$$
\left\|\left(T, T^{*}\right)\right\|_{2, p}=\left(\|T\|^{p}+\left\|T^{*}\right\|^{p}\right)^{1 / p}=2^{1 / p}\|T\|
$$

and by (2.25) we get

$$
\|T\| \leq \delta_{p}(T) \leq 2^{1 / p}\|T\|
$$

for any $T \in B(H)$ and $p \geq 1$.

For $p=2$, we get

$$
\|T\| \leq \delta(T) \leq \sqrt{2}\|T\|
$$

while for $p=1$ we get

$$
\|T\| \leq \delta_{1}(T) \leq 2\|T\|
$$

for any $T \in B(H)$.

From 2.32 we get for $r \geq q \geq 1$ that

$$
\delta_{r}(T) \leq \delta_{q}(T) \leq 2^{\frac{r-q}{r q}} \delta_{r}(T)
$$

for any $T \in B(H)$.

For any $T \in B(H)$ and $p, q>1$ with $\frac{1}{p}+\frac{1}{q}=1$, then by 2.43) we have

$$
\delta_{q}(T) \geq \frac{1}{2^{1 / p}}\left\|T+T^{*}\right\| .
$$

In particular, for $p=q=2$ we get

$$
\delta(T) \geq \frac{\sqrt{2}}{2}\left\|T+T^{*}\right\|,
$$

for any $T \in B(H)$.

By using the inequality 2.45 we get

$$
\delta_{p}(T) \leq \vartheta_{p}(T) \leq 2^{1 / p}\|T\|
$$


for any $T \in B(H)$ and $p \geq 1$.

For $p=1$ we get

$$
\delta_{1}(T) \leq \vartheta_{1}(T) \leq 2\|T\|
$$

for any $T \in B(H)$.

For $p \geq 2$, by employing the equality 2.46 we get

$$
\vartheta_{p}(T)=\left[w_{h, 2, p / 2}\left(|T|^{2},\left|T^{*}\right|^{2}\right)\right]^{1 / 2}=\left[2^{2 / p} w\left(|T|^{2}\right)\right]^{1 / 2}=2^{1 / p}\|T\|
$$

for any $T \in B(H)$.

On utilising (3.7), (3.9) and (3.11) we get

$$
\begin{aligned}
& 0 \leq \delta^{2}(T)-\frac{1}{2} \delta_{1}^{2}(T) \leq \frac{1}{2}\|T\|^{2}, \\
& 0 \leq \delta^{2}(T)-\frac{1}{2} \delta_{1}^{2}(T) \leq\|T\| \delta_{1}(T)
\end{aligned}
$$

and

$$
0 \leq \delta(T)-\frac{1}{\sqrt{2}} \delta_{1}(T) \leq \frac{\sqrt{2}}{4}\|T\|
$$

for any $T \in B(H)$.

Observe, by 4.3 we have that

$$
w_{h, 2, e}\left(\left(T, T^{*}\right)\right)=\sqrt{2} w(T),
$$

for any $T \in B(H)$.

Assume that $T \in B(H)$ and $\gamma, \Gamma \in \mathbb{C}$ with $\Gamma \neq \gamma$ such that

$$
w\left(T-\frac{\gamma+\Gamma}{2} I\right), w\left(T^{*}-\frac{\gamma+\Gamma}{2} I\right) \leq \frac{1}{2}|\Gamma-\gamma|,
$$

then by (3.27) we get

$$
w^{2}(T) \leq\|\operatorname{Re}(T)\|^{2}+\frac{1}{4}|\Gamma-\gamma|^{2},
$$

where $\operatorname{Re}(T):=\frac{T+T^{*}}{2}$.

If $\operatorname{Re}(\Gamma \bar{\gamma})>0$, then by (3.28) and (3.29)

$$
w(T) \leq \frac{1}{2} \frac{|\Gamma+\gamma|}{\sqrt{\operatorname{Re}(\Gamma \bar{\gamma})}}\|\operatorname{Re}(T)\|
$$


and

$$
w^{2}(T) \leq[\|\operatorname{Re}(T)\|+[|\Gamma+\gamma|-2 \sqrt{(\Gamma \bar{\gamma})}]]\|\operatorname{Re}(T)\| .
$$

If $\Gamma \neq-\gamma$, then by 3.30 we get

$$
w(T) \leq\|\operatorname{Re}(T)\|+\frac{1}{8} \frac{|\Gamma-\gamma|^{2}}{|\Gamma+\gamma|} .
$$

Due to the fact that $w(A)=w\left(A^{*}\right)$ for any $A \in B(H)$, the condition 4.19 can be simplified as follows.

If $m, M$ are real numbers with $M>m$ and if

$$
w\left(T-\frac{m+M}{2} I\right) \leq \frac{1}{2}(M-m)
$$

then

$$
w^{2}(T) \leq\|\operatorname{Re}(T)\|^{2}+\frac{1}{4}(M-m)^{2}
$$

If $m>0$, then

$$
w(T) \leq \frac{1}{2} \frac{m+M}{\sqrt{m M}}\|\operatorname{Re}(T)\|
$$

and

$$
w^{2}(T) \leq\left[\|\operatorname{Re}(T)\|+(\sqrt{M}-\sqrt{m})^{2}\right]\|\operatorname{Re}(T)\| .
$$

If $M \neq-m$, then

$$
w(T) \leq\|\operatorname{Re}(T)\|+\frac{1}{8} \frac{(M-m)^{2}}{m+M} .
$$

For other numerical radius and norm inequalities, the interested reader may also consult [1] and [6] and compare these results. The details are not provided here.

\section{INEQUALITIES FOR REAL NORMS}

If $X$ is a complex linear space, then the functional $\|\cdot\|$ is a real norm, if the homogeneity property in the definition of the norms is satisfied only for real numbers, namely we have

$$
\|\alpha x\|=|\alpha|\|x\| \quad \text { for any } \alpha \in \mathbb{R} \text { and } x \in X .
$$


For instance if we consider the complex linear space of complex numbers $\mathbb{C}$ then the functionals

$$
\begin{aligned}
|z|_{p}:=\left(|\operatorname{Re}(z)|^{p}+|\operatorname{Im}(z)|^{p}\right)^{1 / p}, & p \geq 1, \\
|z|_{\infty}:=\max \{|\operatorname{Re}(z)|,|\operatorname{Im}(z)|\}, \quad & p=\infty,
\end{aligned}
$$

are real norms on $\mathbb{C}$.

For $T \in B(H)$ we consider the Cartesian decomposition

$$
T=\operatorname{Re}(T)+i \operatorname{Im}(T)
$$

where the selfadjoint operators $\operatorname{Re}(T)$ and $\operatorname{Im}(T)$ are uniquely defined by

$$
\operatorname{Re}(T)=\frac{T+T^{*}}{2} \quad \text { and } \quad \operatorname{Im}(T)=\frac{T-T^{*}}{2 i} .
$$

We can introduce the following functionals

$$
\|T\|_{r, p}:=\left(\|\operatorname{Re}(T)\|^{p}+\|\operatorname{Im}(T)\|^{p}\right)^{1 / p}, \quad p \geq 1,
$$

and

$$
\|T\|_{r, \infty}:=\max \{\|\operatorname{Re}(T)\|,\|\operatorname{Im}(T)\|\}, \quad p=\infty,
$$

where $\|\cdot\|$ is the usual operator norm on $B(H)$. The definition can be extended for any other norms on $B(H)$ or its subspaces.

Using the properties of the norm $\|\cdot\|$ and the Minkowski's inequality

$$
\left(|a+b|^{p}+|c+d|^{p}\right)^{1 / p} \leq\left(|a|^{p}+|c|^{p}\right)^{1 / p}+\left(|b|^{p}+|d|^{p}\right)^{1 / p}
$$

for $p \geq 1$ and $a, b, c, d \in \mathbb{C}$, we observe that $\|\cdot\|_{r, p}, p \in[1, \infty]$ is a real norm on $B(H)$.

For $p \geq 1$ and $T \in B$ we can introduce the following functionals

$$
\begin{aligned}
\eta_{r, p}(T): & =\sup _{\|x\|=\|y\|=1}\left(|\operatorname{Re}\langle T x, y\rangle|^{p}+|\operatorname{Im}\langle T x, y\rangle|^{p}\right)^{1 / p} \\
& =\sup _{\|x\|=\|y\|=1}\left(|\langle\operatorname{Re} T x, y\rangle|^{p}+|\langle\operatorname{Im} T x, y\rangle|^{p}\right)^{1 / p} \\
& =\|(\operatorname{Re} T, \operatorname{Im} T)\|_{h, 2, p},
\end{aligned}
$$




$$
\begin{aligned}
\theta_{r, p}(T) & :=\sup _{\|x\|=1}\left(|\operatorname{Re}\langle T x, x\rangle|^{p}+|\operatorname{Im}\langle T x, x\rangle|^{p}\right)^{1 / p} \\
& =\sup _{\|x\|=1}\left(|\langle\operatorname{Re} T x, x\rangle|^{p}+|\langle\operatorname{Im} T x, x\rangle|^{p}\right)^{1 / p} \\
& =w_{h, 2, p}(\operatorname{Re} T, \operatorname{Im} T)
\end{aligned}
$$

and

$$
\kappa_{r, p}(T):=\sup _{\|x\|=1}\left(\|\operatorname{Re} T x\|^{p}+\|\operatorname{Im} T x\|^{p}\right)^{1 / p}=\|(\operatorname{Re} T, \operatorname{Im} T)\|_{s, 2, p} .
$$

The case $p=2$ is of interest since for $T \in B(H)$ we have

$$
\begin{aligned}
\eta_{r, 2}(T) & :=\sup _{\|x\|=\|y\|=1}\left(|\operatorname{Re}\langle T x, y\rangle|^{2}+|\operatorname{Im}\langle T x, y\rangle|^{2}\right)^{1 / 2} \\
& =\sup _{\|x\|=\|y\|=1}|\langle T x, y\rangle|=\|T\|, \\
\theta_{r, 2}(T): & =\sup _{\|x\|=1}\left(|\operatorname{Re}\langle T x, x\rangle|^{2}+|\operatorname{Im}\langle T x, x\rangle|^{2}\right)^{1 / 2} \\
& =\sup _{\|x\|=1}|\langle T x, x\rangle|=w(T)
\end{aligned}
$$

and

$$
\begin{aligned}
\kappa_{r, 2}(T) & :=\sup _{\|x\|=1}\left(\|\operatorname{Re} T x\|^{2}+\|\operatorname{Im} T x\|^{2}\right)^{1 / 2} \\
& =\sup _{\|x\|=1}\left(\left\langle(\operatorname{Re} T)^{2} x, x\right\rangle+\left\langle(\operatorname{Im} T)^{2} x, x\right\rangle\right)^{1 / 2} \\
& =\sup _{\|x\|=1}\left(\left\langle\left[(\operatorname{Re} T)^{2}+(\operatorname{Im} T)^{2}\right] x, x\right\rangle\right)^{1 / 2} \\
& =\left\|(\operatorname{Re} T)^{2}+(\operatorname{Im} T)^{2}\right\|^{1 / 2}=\left\|\frac{|T|^{2}+\left|T^{*}\right|^{2}}{2}\right\|^{1 / 2} .
\end{aligned}
$$


For $p=\infty$ we have

$$
\begin{aligned}
\eta_{r, \infty}(T): & =\sup _{\|x\|=\|y\|=1}(\max \{|\operatorname{Re}\langle T x, y\rangle|,|\operatorname{Im}\langle T x, y\rangle|\}) \\
& =\max \left\{\sup _{\|x\|=\|y\|=1}|\langle\operatorname{Re} T x, y\rangle|, \sup _{\|x\|=\|y\|=1}|\langle\operatorname{Im} T x, y\rangle|\right\} \\
& =\max \{\|\operatorname{Re} T\|,\|\operatorname{Im} T\|\},
\end{aligned}
$$

and in a similar way

$$
\theta_{r, \infty}(T)=\kappa_{r, \infty}(T)=\max \{\|\operatorname{Re} T\|,\|\operatorname{Im} T\|\}=\|T\|_{r, \infty} .
$$

The functionals $\eta_{r, p}, \theta_{r, p}$ and $\kappa_{r, p}$ with $p \in[1, \infty]$ are real norms on $B(H)$.

We have

$$
\begin{aligned}
\eta_{r, p}(T) & =\sup _{\|x\|=\|y\|=1}\left(|\operatorname{Re}\langle T x, y\rangle|^{p}+|\operatorname{Im}\langle T x, y\rangle|^{p}\right)^{1 / p} \\
& \leq\left(\sup _{\|x\|=\|y\|=1}|\operatorname{Re}\langle T x, y\rangle|^{p}+\sup _{\|x\|=\|y\|=1}|\operatorname{Im}\langle T x, y\rangle|^{p}\right)^{1 / p} \\
& =\left(\|\operatorname{Re}(T)\|^{p}+\|\operatorname{Im}(T)\|^{p}\right)^{1 / p}=\|T\|_{r, p}
\end{aligned}
$$

and

$$
\begin{aligned}
\|T\|_{r, \infty} & =\sup _{\|x\|=\|y\|=1}(\max \{|\operatorname{Re}\langle T x, y\rangle|,|\operatorname{Im}\langle T x, y\rangle|\}) \\
& \leq \sup _{\|x\|=\|y\|=1}\left(|\operatorname{Re}\langle T x, y\rangle|^{p}+|\operatorname{Im}\langle T x, y\rangle|^{p}\right)^{1 / p}=\eta_{r, p}(T)
\end{aligned}
$$

for any $p \geq 1$ and $T \in B(H)$.

In a similar way we have

$$
\|T\|_{r, \infty} \leq \theta_{r, p}(T) \leq\|T\|_{r, p}
$$

and

$$
\|T\|_{r, \infty} \leq \kappa_{r, p}(T) \leq\|T\|_{r, p}
$$

for any $p \geq 1$ and $T \in B(H)$. 
If we write the inequality $(1.13)$ for $n=2, T_{1}=\operatorname{Re} T$ and $T_{2}=\operatorname{Im} T$ then we get

$$
\theta_{r, p}(T) \leq \eta_{r, p}(T) \leq 2 \theta_{r, p}(T)
$$

for any $p \geq 1$ and $T \in B(H)$.

Using the inequalities (2.25) and (2.26) for $n=2, T_{1}=\operatorname{Re} T$ and $T_{2}=\operatorname{Im} T$ then we get

$$
\frac{1}{2^{1 / p}}\|T\|_{r, p} \leq \eta_{r, p}(T) \leq\|T\|_{r, p}
$$

and

$$
\frac{1}{2^{1 / p}}\|T\|_{r, p} \leq \theta_{r, p}(T) \leq\|T\|_{r, p}
$$

for any $p \geq 1$ and $T \in B(H)$.

If we use the inequalities 2.32 and 2.33 for $n=2, T_{1}=\operatorname{Re} T$ and $T_{2}=\operatorname{Im} T$ then we get for $t \geq p \geq 1$ that

$$
\eta_{r, t}(T) \leq \eta_{r, p}(T) \leq 2^{\frac{t-p}{t p}} \eta_{r, t}(T)
$$

and

$$
\theta_{r, t}(T) \leq \theta_{r, p}(T) \leq 2^{\frac{t-p}{t p}} \theta_{r, t}(T)
$$

for any $T \in B(H)$.

For $p=1$ we have the functionals

$$
\begin{gathered}
\eta_{r, 1}(T)=\sup _{\|x\|=\|y\|=1}(|\langle\operatorname{Re} T x, y\rangle|+|\langle\operatorname{Im} T x, y\rangle|)=\|(\operatorname{Re} T, \operatorname{Im} T)\|_{h, 2,1}, \\
\theta_{r, 1}(T):=\sup _{\|x\|=1}(|\langle\operatorname{Re} T x, x\rangle|+|\langle\operatorname{Im} T x, x\rangle|)=w_{h, 2,1}(\operatorname{Re} T, \operatorname{Im} T)
\end{gathered}
$$

and

$$
\kappa_{r, 1}(T):=\sup _{\|x\|=1}(\|\operatorname{Re} T x\|+\|\operatorname{Im} T x\|)=\|(\operatorname{Re} T, \operatorname{Im} T)\|_{s, 2,1} .
$$

By utilising the inequalities (3.7), (3.9) and 3.11) for $n=2, T_{1}=\operatorname{Re} T$ and $T_{2}=\operatorname{Im} T$, then

$$
\begin{aligned}
& 0 \leq\|T\|^{2}-\frac{1}{2} \eta_{r, 1}^{2}(T) \leq \frac{1}{2}(\max \{\|\operatorname{Re} T\|,\|\operatorname{Im} T\|\})^{2} \\
& 0 \leq\|T\|^{2}-\frac{1}{2} \eta_{r, 1}^{2}(T) \leq \max \{\|\operatorname{Re} T\|,\|\operatorname{Im} T\|\} \eta_{r, 1}(T)
\end{aligned}
$$


and

$$
0 \leq\|T\|-\frac{\sqrt{2}}{2} \eta_{r, 1}(T) \leq \frac{\sqrt{2}}{4} \max \{\|\operatorname{Re} T\|,\|\operatorname{Im} T\|\}
$$

for any $T \in B(H)$.

Also, by utilising the inequalities (3.8), (3.10) and (3.12) for $n=2, T_{1}=$ $\operatorname{Re} T$ and $T_{2}=\operatorname{Im} T$, then

$$
\begin{aligned}
& 0 \leq w^{2}(T)-\frac{1}{2} \theta_{r, 1}^{2}(T) \leq \frac{1}{2}(\max \{\|\operatorname{Re} T\|,\|\operatorname{Im} T\|\})^{2} \\
& 0 \leq w^{2}(T)-\frac{1}{2} \theta_{r, 1}^{2}(T) \leq \max \{\|\operatorname{Re} T\|,\|\operatorname{Im} T\|\} \theta_{r, 1}(T)
\end{aligned}
$$

and

$$
0 \leq w(T)-\frac{\sqrt{2}}{2} \theta_{r, 1}(T) \leq \frac{\sqrt{2}}{4} \max \{\|\operatorname{Re} T\|,\|\operatorname{Im} T\|\}
$$

for any $T \in B(H)$.

If $m, M$ are real numbers with $M>m$ and if

$$
\left\|\operatorname{Re} T-\frac{m+M}{2} I\right\|,\left\|\operatorname{Im} T-\frac{m+M}{2} I\right\| \leq \frac{1}{2}(M-m),
$$

then by 3.27 we get

$$
w^{2}(T) \leq \frac{1}{2}\|\operatorname{Re} T+\operatorname{Im} T\|^{2}+\frac{1}{2}(M-m)^{2} .
$$

If $m>0$, then $(3.28)$ and $(3.29)$ we have

$$
w(T) \leq \frac{1}{2 \sqrt{2}} \frac{m+M}{\sqrt{m M}}\|\operatorname{Re} T+\operatorname{Im} T\|
$$

and

$$
w^{2}(T) \leq\left[\frac{1}{2}\|\operatorname{Re} T+\operatorname{Im} T\|+(\sqrt{M}-\sqrt{m})^{2}\right]\|\operatorname{Re} T+\operatorname{Im} T\| .
$$

If $M \neq-m$, then by 3.30 we get

$$
w(T) \leq \frac{1}{\sqrt{2}}\left(\|\operatorname{Re} T+\operatorname{Im} T\|+\frac{1}{4} \frac{(M-m)^{2}}{M+m}\right) .
$$

Finally, we observe that a simple sufficient condition for $(5.12)$ to hold, is that

$$
m I \leq \operatorname{Re} T, \quad \operatorname{Im} T \leq M I
$$

in the operator order of $B(H)$. 


\section{ACKNOWLEDGEMENTS}

The author would like to thank the anonymous referee for valuable suggestions that have been implemented in the final version of the paper.

\section{REFERENCES}

[1] M. BAKHERAD, K. SheBRAWI, Upper bounds for numerical radius inequalities involving off-diagonal operator matrices, Ann. Funct. Anal. 9 (3) (2018), $297-309$.

[2] S.S. Dragomir, A counterpart of Schwarz's inequality in inner product spaces, East Asian Math. J. 20 (1) (2004), 1-10, preprint RGMIA Res. Rep. Coll. 6 (2003), http://rgmia.org/papers/v6e/CSIIPS.pdf.

[3] S.S. Dragomir, A survey on Cauchy-Bunyakovsky-Schwarz type discrete inequalities, J. Inequal. Pure Appl. Math. 4 (3) (2003), Article 63, 142 pp., https://www.emis.de/journals/JIPAM/article301.html?sid=301.

[4] S.S. Dragomir, "Advances in Inequalities of the Schwarz, Grüss and Bessel Type in Inner Product Spaces", Nova Science Publishers Inc., NY, 2005.

[5] S.S. Dragomir, The hypo-Euclidean norm of an $n$-tuple of vectors in inner product spaces and applications, J. Ineq. Pure E6 Appl. Math. 8(2) (2007), Article 52, 22 pp., https://www.emis.de/journals/JIPAM/article854.html?sid=854

[6] S.S. Dragomir, Some inequalities for the Euclidean operator radius of two operators in Hilbert spaces, Linear Algebra Appl. 419 (1) (2006), 256-264.

[7] S.S. Dragomir, Reverses of the Schwarz inequality generalising the KlamkinMcLeneghan result, Bull. Austral. Math. Soc. 73 (1) (2006), 69-78.

[8] S. S. Dragomir, Some inequalities of Kato type for sequences of operators in Hilbert spaces, Publ. Res. Inst. Math. Sci. 48 (4) (2012), 937-955.

[9] S. S. Dragomir, Y. J. Cho, Y.-H. Kim, Applications of Kato's inequality for $n$-tuples of operators in Hilbert spaces, (I), J. Inequal. Appl. 2013:21 (2013), 16pp.

[10] S. S. Dragomir, Y. J. Cho, Y.-H. Kim, Applications of Kato's inequality for $n$-tuples of operators in Hilbert spaces, (II), J. Inequal. Appl. 2013:464 (2013), 20 pp.

[11] T. Kato, Notes on some inequalities for linear operators, Math. Ann. 125 (1952), $208-212$.

[12] M.S. Moslehian, M. Sattari, K. Shebrawi, Extensions of Euclidean operator radius inequalitie, Math. Scand. 120 (1) (2017), 129-144.

[13] G. Popescu, "Unitary Invariants in Multivariable Operator Theory", Mem. Amer. Math. Soc. no. 941, 2009.

[14] A. Sheikhhosseini, M.S. Moslehian, K. Shebrawi, Inequalities for generalized Euclidean operator radius via Young's inequality. J. Math. Anal. Appl. 445 (2) (2017), 1516-1529.

[15] O. Shisha, B. Mond, Bounds on differences of means, in "Inequalities", Academic Press Inc., New York, 1967, 293-308. 